\title{
Collaborative Advanced Building Methodology toward Industrialization of Informal Settlements in Cairo
}

\author{
Camilla Follini", Rongbo Hu', Wen Pan', Thomas Linnera, and Thomas Bock \\ ${ }^{a}$ Chair for Building Realisation and Robotics, Technical University of Munich, Germany \\ E-mail: camilla.follini@tum.de
}

\begin{abstract}
-
Today's population has reached a growth rate never seen before, and, globally, there are examples of traditional cities that have failed to adapt to this sudden change. Egypt makes no exception in this trend. In its largest city Cairo, this trend caused a considerable expansion of the phenomenon of informal settlements, areas spontaneously built by the inhabitants, often lacking regulation, planning, and basic needs. By investigating the context of Cairo, in particular the area of Sakiat Mekki, the paper explores the possibility of improving the quality of life in informal settlements with sustainable decentralized processing units (DPUs), tailored to an affordable and adaptable building system $\left(A^{2} B S\right)$. The goal of the DPUs is to enhance three main aspects of life (working, energy, and mobility) by gradually implementing small-scale subsystems of advanced construction in the hosting environment, thus providing a more flexible solution to evolve in time. The design was developed after an analysis based on Requirements Engineering (RE), to ensure that the system would be more easily accepted and the design proactively influenced by the existing community's feedback. Accordingly, the construction elements were designed based on stakeholders' needs. Thanks to a simplified construction process and joining system, not only this solution can be easily assembled by unskilled labor, but it could also be used as a step forward toward automation of the building process in substandard environments.
\end{abstract}

Keywords -

Collaborative design and construction; Industrialization of informal settlements; Decentralized Processing Unit; Affordable and Adaptable Building System

\section{Introduction}

Egypt has been heavily influenced during the last decades by economic, social and political pressures.
Cities such as Cairo, which are the focus of the government and the society, are facing great population growth, immigration growth from villages to big cities and immigration of refugees. Like many mega cities of developing countries, Cairo has experienced its strongest growth in the half century after World War II. The strongest growth has been registered between 1996 and 2006, with a population increase of about $4,000,000$ people. More recent data show that the growth is steadily slowing down. Still, these numbers are high enough to raise concerns about if and how the government had planned for the sustainable housing of the additional inhabitants [1]. The problem of informal settlements is considered one of the most challenging issues in the developing world, since it poses an economic and social threat to countries' future. Most of informal settlements share some distinctive traits, like poor structural quality of housing and infrastructure, lack of basic services, such as water, sanitation, waste collection, schools, hospitals, and poor environmental conditions. However, the nature of informal settlements also could impose some advantages and benefits. There are many aspects, which city planner, architects and environmental agencies could adopt: 1) self-sufficiency, as shops and local produce are vastly available, 2) short walking distance to reach the workplace and most of the amenities, 3) strong sense of community, 4) enhanced safety, since neighborhood connection is very close and people tend to help each other more willingly, and 5) sense of participation, as most of the services, such as street maintenance and garbage collection, are effectively controlled by the inhabitants themselves [2].

\section{Methods}

Given the broad scope of the objectives goal, a systematic method has been used to determine the best approach and methodology. This chapter presents how the project is conducted.

\subsection{Scope and Approach}

Considering the advantages and drawbacks of the 
current situation stated above, the aim of the $\mathrm{A}^{2} \mathrm{~L}$ Mobilius project [3] is to develop modular, adaptable and scalable units, which responds to future socio, economic, technological and environmental change, by exploring the Egyptian context and investigating the possibility and requirements for an efficient infrastructure. In order to achieve this, the research has been focused on two main elements:

1. Affordable and Adaptable Building System (A $\left.{ }^{2} \mathrm{BS}\right)$ : a flexible building kit composed of prefabricated elements. The kit has been designed to fit the informal environment, and adapt through time to the community change. Its goal is to gradually replace informal structures and thus "formalize" the built environment.

2. Decentralized Processing Unit (DPU): a prefabricated, self-sustaining, interchangeable, and standardized system that houses a sum of technological equipment needed for a household. It would allow for a step by step upgrade of the informal settlement.

The decentralized processing unit has been divided into three sub-systems, basing on the most relevant issues of the hosting country: energy, mobility and lifework balance [4]. The energy subsystem is meant to provide a reliable structure that would respond to the needs of the community related to collection, provision, wise use and eventual production of different kind of primary resources such as electricity, gas and water. The mobility subsystem addresses external mobility and the optimization of transportation of goods and people. Finally, the home fabrication subsystem is part of a greater interconnected system aiming to give an alternative and more sustainable work method, and therefore to provide a higher number of workplaces to decrease the unemployment rate. The decentralized processing unit elements will be integrated to the building kit, to form a comprehensive system.

\subsection{Overall Methodology}

The methodology for the project plan has been based on the V-Model diagram. The project has been divided into smaller problems to be addressed with one design module or sum of modules. As show in Figure 1, the steps of the model comprehend on or more work packages of the project. The left side of the scheme shows the preparatory work or project definition, which starts from a general perspective that is detailed in time. On the opposite, the project implementation, on the right side starts from the detailing of modules to reach a general system in the later stages. As it can be also seen from the diagram, the system is set to go through several iteration loops for optimization. Two cycles in particular concern communication with stakeholders, one to verify the premise of the project, thus the requirement analysis, and the second to verify the design. Final validation will be given at the end of the project, with the comparison of the final system and the revised requirement and stakeholder analysis. The current paper presents the first part of the implementation section, in between the two verification loops, as highlighted with the black square in the diagram. The modules of the main elements have been established basing on the requirement analysis. They will then be tested in a system. Therefore, the material presented in this paper is to be considered part of a conceptual stage to be further optimized and validated thanks to real world feedback.

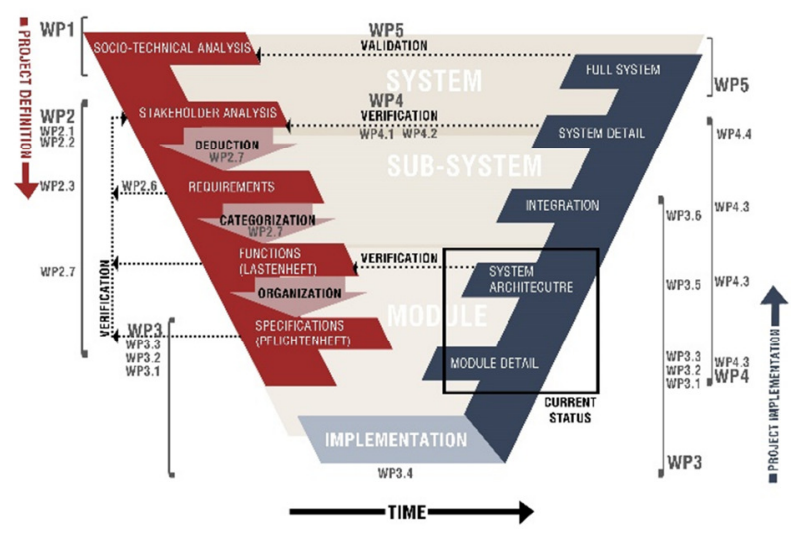

Figure 1: V-Model of the $\mathrm{A}^{2} \mathrm{~L}-$ Mobilius project

\subsection{Requirement Analysis}

Collaboration with the final users is generally essential for the success of any project aimed to the revitalization of informal settlements. Therefore, participation and feedback of stakeholders throughout the project is a critical factor to be ensured. Thus, a methodology based on Requirements Engineering (RE) has been first developed, in order to find the most fitting solutions based on environmental requirements and stakeholders wishes [5]. The structure of the methodology takes into consideration repetitive optimization loops to constantly monitor and optimize the stakeholder and requirement analysis for the whole duration of the project. The aim of the requirement analysis, together with a technological feasibility study, was to identify concrete functions to be translated into design elements, and a suitable system architecture to give them a common structure. The output of the analysis carried out for the three DPU subsystems is shown in Figure 2. 

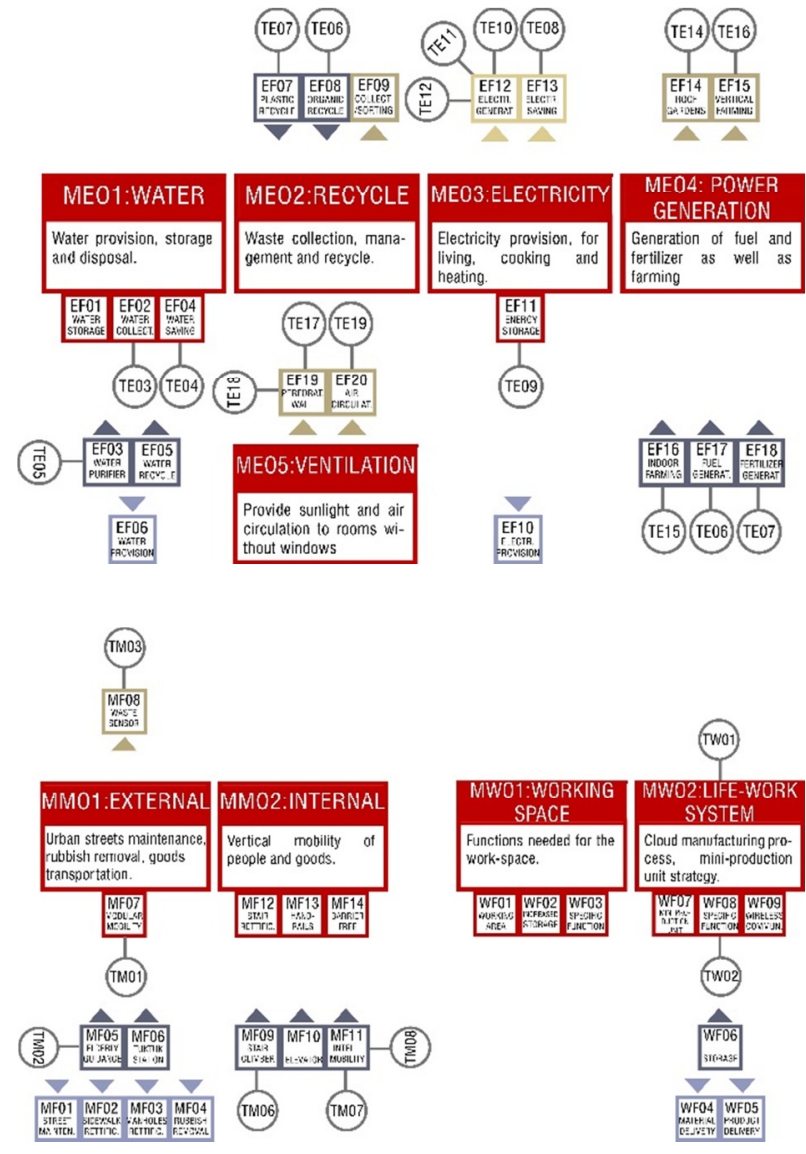

Figure 2: Requirement and function analysis of the three DPU subsystems (top: energy, bottom left: mobility and right: working)

\subsection{Technology Transfer from High to Low- tech Environments}

There are a wide range of research topics in the area of integrating new technologies into the living environment (buildings, city, infrastructure, design). The research projects below represent the examples of technologies in regard to three major aspects (working, energy, and mobility subsystems). As a matter of fact, it is a challenge in this project to transfer these approaches into the Egyptian environment. To achieve that goal, basic strategy and approach shall be retained yet in a more energy-efficient and cost-effective way in order to fit into Egyptian context, such as simplifying the design, using local materials, and adapting to the culture. After successfully implementing these technologies in the developing environment, a strategy of reverse innovation could be adopted in order that these improved technologies can be reintroduced to the industrialized world, thus not only benefiting the local residents, but also the developed countries. For example, the company GE Healthcare is selling an ultraportable electrocardiograph machine in the U.S. at a
$20 \%$ cost for similar devices, which is originally developed for markets in India and China [6].

\subsubsection{Working Subsystem}

Extensive research has been carried out in the topic of living in the future, as well as in the topic of decentralized production in modular mini-factories. For instance, in the project $\mathrm{USA}^{2}$ [7], assisted workspaces for decentralized high-tech home production of customized goods have been developed by Chair of Building Realisation and Robotics at TUM $\left(\mathrm{BR}^{2}\right)$. The research project, financed by the German Federal Ministry of Education and Research (BMBF, grant number: 16SV6191) within the Human-Technology Interaction (MTI) program, concerned the creation of workspaces embedded in larger Cloud Manufacturing systems, which will allow companies to utilize a highly skilled workforce (including highly experienced elderly) worldwide. Within the project, the workspace (its functions and design), the embedded sensors distributed in the environments, novel interfaces (such as leap motion sensors [8]) and the related work processed were fully co-adapted (Figure 3 ). The $\mathrm{USA}^{2}$ showed that the co-adaptation of environment and mobile platform allows for a multitude of assistive functions, such as intuitive user interface, transports of goods, and detection of emergencies. Considering the high level of participation and self-organization already existing in informal settlements, a suitable strategy for building prefabrication could be the organization of a collaborative manufacturing decentralized structure. Instead of providing a central factory where material is produced in mass quantity, several small manufacturing stations can be located all over the settlement to work as one. In the same direction, the project $\mathrm{USA}^{2}$ offers an interesting example of Mini-Production Unit (MPU) developed to be embedded in a cloud-manufacturing system.

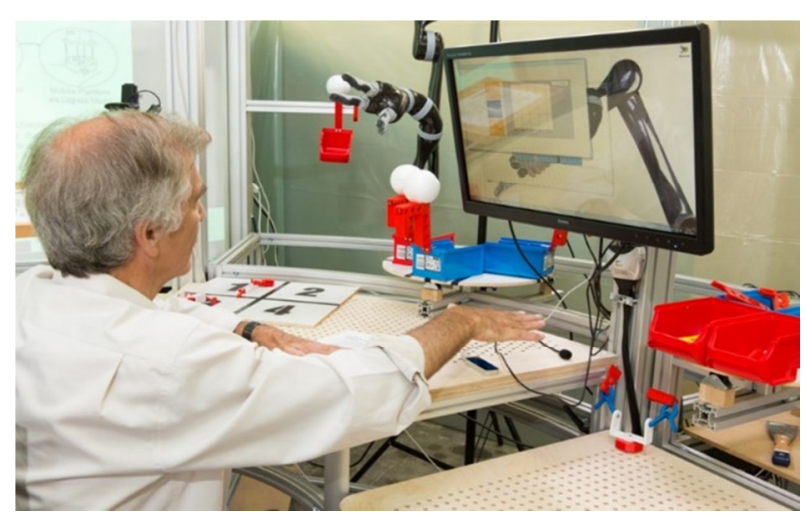

Figure 3: $\mathrm{USA}^{2}$ mini producion unit (Image: Schlegelmilch) 


\subsubsection{Energy Subsystem}

Energy saving and prefabrication in building renovation are two of the main goals of the BERTIM Project [9], developed in a large consortium $\mathrm{BR}^{2}$ is a partner of, which has received funding from the European Union's Horizon 2020 Research and Innovation Program under grant agreement No. 636984 (Figure 4). The aim of the project is to develop [10]:

1. Prefabricated modules for renovation, already equipped with architectural elements and technologies for reduction of energy consumption (such as windows, insulation materials, collective HVAC systems, renewable energy systems and energy supply systems). The panels are designed to be easily installed and they are produced with low carbon foot print and materials that can be recycled after the use stage.

2. An innovative methodology for the renovation process, making use of the latest software technologies such as BIM and CAD/CAM tools

3. Business opportunities for professionals in the renovation sector.

To increase the feasibility of the project, the renovation process, as well as the timber prefabricated modules have been planned to be validated in two actual buildings located in two different climatic regions of Europe (north and south) [11].

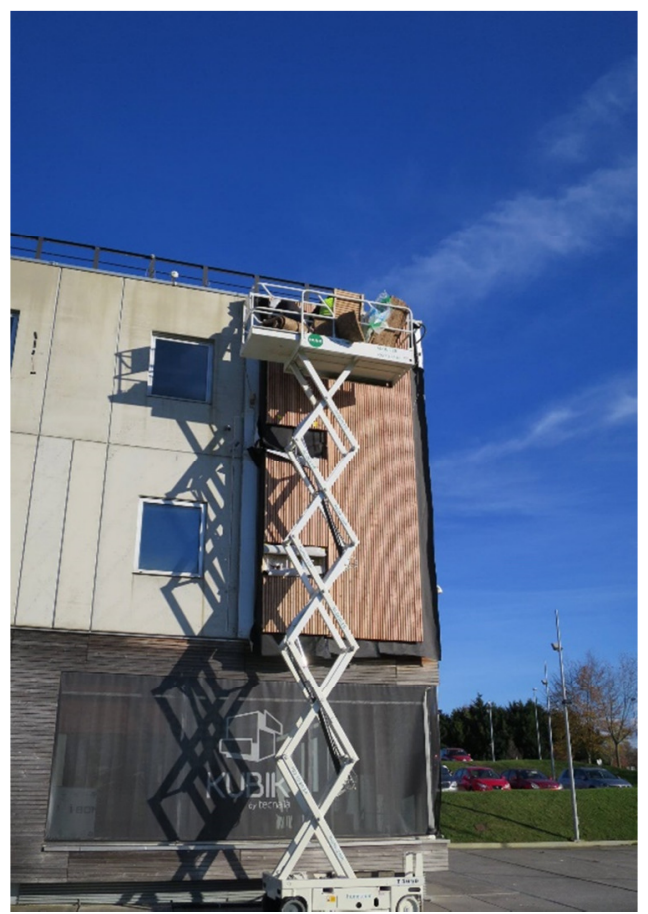

Figure 4: BERTIM facade modules installation in one of the sites (Image: M. Eng. Kepa Iturralde).

\subsubsection{Mobility Subsystem}

The abbreviation of the Horizon 2020 project REACH stands for Responsive Engagement of the Elderly Promoting Activity and Customized Healthcare [12]. REACH represents a solution that seeks to reduce Long Term Care (LTC) by serving as a personalized system for promoting and monitoring the activity of elderly citizens in order to reduce their risk of function loss and related morbidities. During the four years' duration of the project, the consortium develops modular, personalized medical and ethical acceptable solutions integrated in and around buildings that allow an intelligent prediction about the health status of people/patients. Based on forecast and analytic algorithms, REACH will be able to provide novel, personalized interventions such as customized services, products, and equipment for mobilization and rehabilitation, physical activity, training, food and nutrition, mobility, and patient motivation.

PASSAge project, which is financed by the German Federal Ministry of Research and Education (BMBF), under the Grant Number V4MOB059, is another example showing this approach [13]. In PASSAge a mobile robot was developed that could flexibly couple to various mobility devices (rollators, wheelchairs, etc.) and thus assist and facilitate the mobility of elderly people (Figure 5).

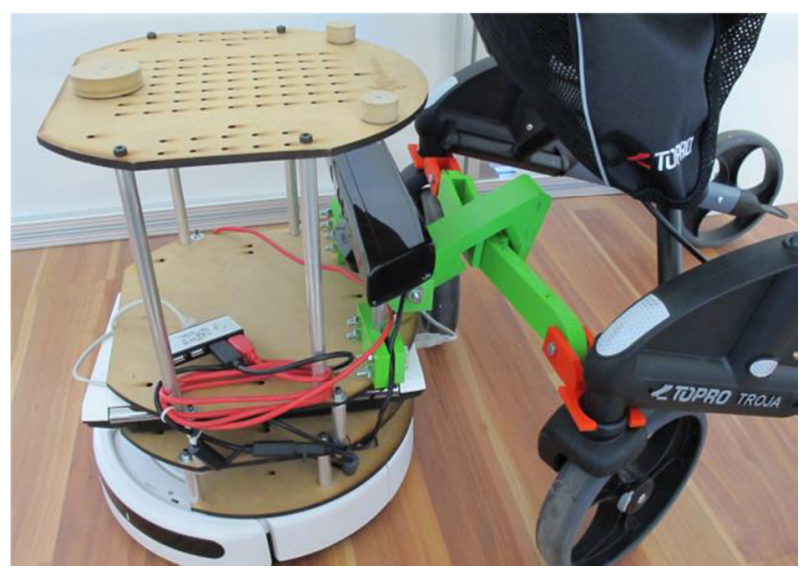

Figure 5: Prototype developed in the project PASSAge (Image: Chair of Building Realisation and Robotics, TUM).

\section{Results}

The system is based on a study case surveyed by the Egyptian partner that is located in the area of Sakiat Mekki, on the west side of the Nile in the Giza district. The building has many problems common to informal settlements, such as lack of space, sanitation issues, lack of ventilation, hazardous internal mobility (i.e. stairs without handrail and different height of risers), and poor 
insulation. The surroundings are as well problematic, the main issues being the poor street maintenance, large presence of uncollected waste, and extremely compact building presence, making it impossible to expand the study case building on the side (Figure 6).
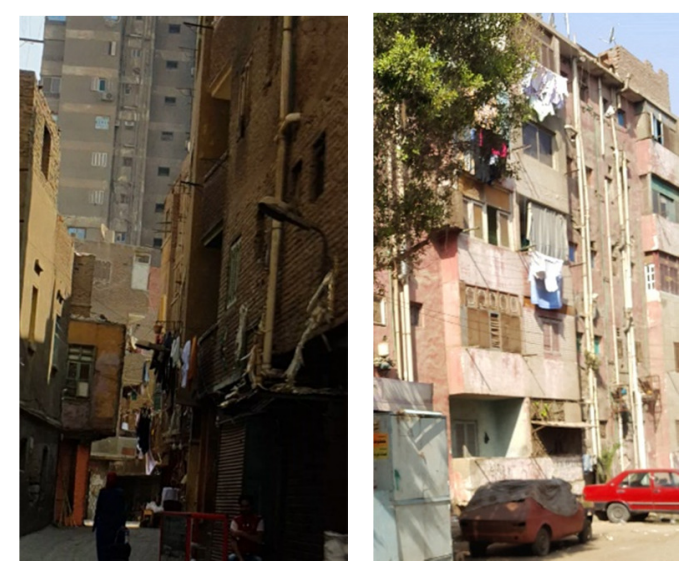

Figure 6: Low sanitation conditions in Farahat street (left) and $6^{\text {th }}$ October street (right) (Image: M. Sc. Maged Helal).

\subsection{Proposed Construction System}

The construction system is based on the modular structure and infill strategy. The panelized system shown in Figure 7 is composed of four modular panels and vertical support pillars. Elements are connected by bolted joints and have a limited dimension for better transportation. The modular infill system is made of a framework that can accommodate different functionalities [14].

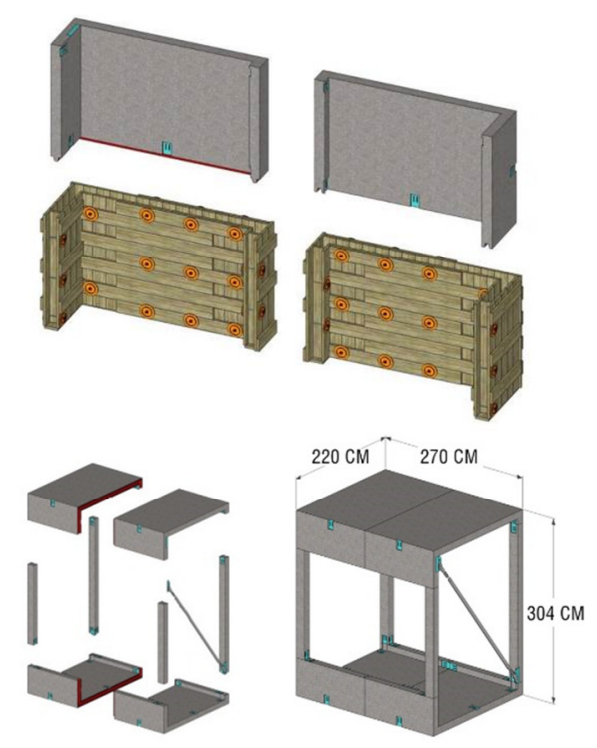

Figure 7: Panel system of the construction kit

\subsection{Decentralized Processing Unit}

As aforementioned, the most important functions and their system architecture have been highlighted thanks to a first analysis based on requirements engineering. The dimensioning of the construction system, carried out in the previous section, was a necessary prior step necessary to translate functions into a suitable design. The geometries considered for the decentralized processing unit are tailored to the $\mathrm{A}^{2} \mathrm{BS}$ module. Each element has been categorized, considering its nature, size and priority, in three classes. Since the final system will be modular, they can be explained as such:

1. Core functions: elements that have high importance to the system and should be present in every configuration. They can be part of the main body (the "chassis") of the module, to which other functions can be attached.

2. Optional functions: functions whose presence is not critical to the operation of the system and can be thus added later in time.

3. External functions: physical elements or services that have to be located or provided from outside of the main body of the module.

\subsubsection{Life-Work Patterns Subsystem}

The home fabrication subsystem has been considered as a part of a greater system, whose goal is to give an alternative and more sustainable work method and provide a higher number of workplaces in order to decrease the unemployment rate, following the example of the project $\mathrm{USA}^{2}$ given in section 2.4.1. Therefore, instead of having a conventional centralized production plant, workspaces are scattered throughout the settlement. Manufacturing models, such as cloud manufacturing, concern the creation of complex objects through a net of professionals and clients working together with wireless communication, narrowing the need of mobility only to material and products. Thus, this model could have the advantage of reducing the overall traffic congestion and of reduce the commuting time, by overlapping work and life spaces. Moreover, the subsystem would assure as well a better, healthier, safer working condition. Given this premises, the model may represent an interesting future development for informal settlements, responding to several of their most relevant problems. Nevertheless, the technology should be adapted to the environment and it should be first proven on the field. Therefore, the team focused on cheap technologies and simple products. A validation of this first proposal will be given by the stakeholders in the next stage.

Among the different products to manufacture, the team focused on an innovative technique based on 
Mycotechnology, which brings a new material into the construction field characterized by a fairly low construction cost and low environmental impact [15]. By using this technology, the aim of the working subsystem is to produce cheap and sustainable construction elements, such as bricks, to be used as part of the construction system. The simple shape and production method permits a simplification of the information flow, compared to more technologically advanced examples such as the $\mathrm{USA}^{2}$ project, to increase the feasibility of the system.

\subsubsection{Energy Subsystem}

The energy subsystem should provide a reliable system that would respond to the needs of the community related to collection, provision, wise use and eventual production of different kind of primary resources such as electricity, gas and water. During the requirement analysis, the functions of the energy subsystem have been sorted into five main clusters, based on their scope: water, recycle, electricity, power generation, and ventilation. Clusters contain one or more DPU modules. Each cluster has its own principle of function, affecting the issues determined during the requirement analysis. In line with the idea of technology transfer, the prefabricated façade modules from BERTIM, introduced in section 2.4.2, have been used as reference for the vertical wall elements, however simplified to adapt to the final context. Moreover, the panel has been tailored to be produced with materials that are cheaper and locally sustainable. In order to propose feasible functions for the Cairene environments, some technologies are a re-reading of traditional techniques. For instance, rainwater harvest, a technology whose first examples in the country date back to at least 2000 years [16], is the main principle of the water cluster. Another element that has been used into the façade module is the Mashrabiya, traditionally used to control light and air direction, reduce temperature, and provide privacy. For ventilation, a possible addition of a wind tower has been considered.

\subsubsection{Mobility Subsystem}

The mobility subsystem should reduce the problems related to people ambulation and goods logistic both outdoors and indoors. Internal mobility is a common problem in informal settlements, due to the fact that apartments are built with no reference to the legal code. The problem of barrier-free design is therefore vast. In fact, even if the new apartments built with the construction system here proposed would fall within the barrier-free category, they would not solve the problem, being the rest of the environment not developed in that direction. Therefore, the approach proposed for the mobility subsystem, especially the one applying to the existing environment, is a gradual modification of the interior. That reconnects to the initial meaning of decentralized processing unit cells, aiming to "infect" the environment and gradually upgrade it. Again, the technologies shown in section 2.4.3 have been considered to be transferred in the subsystem. In particular, the team focused on solutions not heavily relying on hardware elements, such as the falling detection system.

\section{Conclusion and Future Work}

The feasibility of the system will be simulated in three scenarios with different level of diffusion of the two main construction elements (i.e. $\mathrm{A}^{2} \mathrm{BS}$ and DPU) into the selected building (Figure 8). Each level comports different building connotations, which can be summarized in three points. The first aspect is the system standardization. The elements of the building kit have been standardized for easy production and the production units accordingly designed to fit in the kit. Nonetheless, some decentralized processing unit elements have been designed to adapt to an existing environment, in order to increase the flexibility of the system. This brings to the following consideration: the more extensively the construction kit is used, the more the system can be standardized. The second aspect is centralization. Diffusion of decentralized processing unit elements does not necessarily mean that they are physically spread apart. A construction-kit cell can confine the production-unit elements in a specific area of the building and still contain a bigger number of them than an upgraded existing environment. However, in the hypothesis that the construction kit would be used for the entire house, then the decentralized processing unit elements could be eventually embedded throughout the entire construction, and the decentralized processing unit considered ubiquitous.

As highlighted in section 2.2, the research presented in this paper is contextualized in between the two main verification loops based on the V-Model organization strategy. In order to make the verification more accessible, the developed solutions will be contextualized into the study case and divided into easily recognizable aspects by the scenarios. Thereafter they will be ready to go through a final evaluation of relevant stakeholders located in Egypt. The next research step will be to optimize the system according to the stakeholders' feedback, to be best accepted by the end-users. Thanks to a simplified construction process and joining system, not only this solution can be easily assembled by unskilled labor, but it could also be used as a step forward toward automation of the building process in informal settlements. 


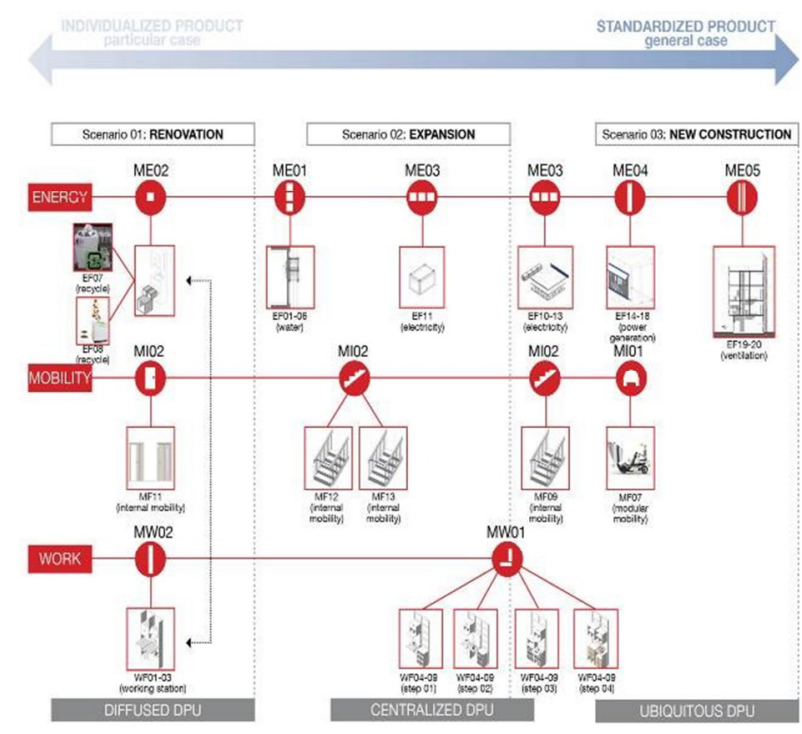

Figure 8: Scenario definition

\section{Acknowledgements}

This research is partly funded by the German Federal Ministry of Education and Research (Project: AL²MOBILIUS; Grant Number: GERF-IB-033 Almobilius_01DH14003). The authors would like to thank the Egyptian partner Dr. Wafaa Nadim at the German University in Cairo for her collaboration and advice. Furthermore, the authors are grateful to $\mathrm{Mr}$. Maged Helal for his support.

\section{References}

[1] Cox W. The Evolving Urban Form: Cairo. Online: http://www.newgeography.com/content/002901the-evolving-urban-form-cairo, Accessed: 08/03/2017.

[2] Helal M. Transformation of informal settlements in Egypt into productive city entities by utilizing and adapting advanced technologies. M.Sc. Thesis. Technical University of Munich, Germany, 2016.

[3] A 2 L-Mobilius project website. Online: http://www.br2.ar.tum.de/index.php/research/proje cts/8-home/167-a2l-mobilius, Accessed: 01/03/2017.

[4] Nadim W. and Bock T. Technological implants for sustainable autonomous upgrading of informal settlements in Cairo-Egypt. In Proceedings of World Sustainable Building Conference, Barcelona, Spain, 2014.

[5] Follini C. Pan W. Linner T. Nadim W. and Bock T. Development of a Methodology based on Requirements Engineering for Informal Settlements upgrading in Cairo. In Proceedings of the CIBW119CIC2016 workshop, pages 45-51, Munich, Germany, 2016.

[6] Jana R. Innovation Trickles in a New Direction. Online:

https://www.bloomberg.com/news/articles/200903-11/innovation-trickles-in-a-new-direction, Accessed: 06/03/2017.

[7] Linner T. Güttler J. Georgoulas C. Zirk A. Schulze E. and Bock T. Development and Evaluation of an Assistive Workstation for Cloud Manufacturing in an Aging Society. In Proceedings of Ambient Assisted Living: 8th AAL-Kongress, Frankfurt, Germany, 2015.

[8] Bassily D. Georgoulas C. Güttler J. Linner T. and Bock T. Intuitive and Adaptive Robotic Arm Manipulation using the Leap Motion Controller. In Proceedings of International Symposium on Robotics ISR ROBOTIK, Munich, Germany, 2014.

[9] BERTIM project website. Online: http://www.bertim.eu, Accessed: 01/03/2017.

[10] European Union Open Data Portal. Funded Projects under Horizon 2020. Secure, clean and efficient energy. Online: https://www.nksenergie.de/lw_resource/datapool/_items/item_337/ nks-e_projects_funded_2014_ee.pdf, Accessed: 23/03/2017.

[11] Iturralde K. Linner T. and Bock T. Development of a modular and integrated productmanufacturing-installation system kit for the automation of the refurbishment process in the research project BERTIM. In proceedings of International Symposium on Automation and Robotics in Construction (ISARC), Auburn, USA, 2016.

[12] REACH project website. Online: http://reach2020.eu, Accessed: 01/03/2017.

[13] Bock T. Güttler J. Georgoulas C. and Linner T. The Development of Intra-House Mobility, Logistics and Transfer Solutions in PASSAge. Journal of Robotics and Mechatronics, Vol.27 No.1, 2015.

[14] Pan W. and Bock T. Development of interactive cluster for urban development in Egypt. In Proceedings of Sustainable Built Environment (SBE), Cairo, Egypt, 2016.

[15] Travaglini S. Noble J. Ross P. G. and Dharan C. K. H. Mycology Matrix Composite. In Proceedings of American Society for Composites, $28^{\text {th }}$ Technical Conference, State College, United states, 2013.

[16] Abdel-Shafy H. I. El-Saharty A. A. Regelsberger M. and Platzer C. Rainwater in Egypt: quantity, distribution and harvesting. Mediterranean Marine Science, 11(2): 245-257, 2010. 